\title{
Heisenberg's uncertain legacy
}

The UK premiere of Simon Stephens's play Heisenberg: The Uncertainty Principle is a reminder that the cultural cachet of Werner Heisenberg's discovery 90 years ago $^{1}$ is as strong as ever. Physics is in fact notable only by its absence in Stephens's play, which is about an unlikely relationship that sparks between the production's two sole characters in a starkly minimalist setting. But the rather tenuous evocation of this tenet of quantum mechanics illustrates how its interpretation in terms of the unpredictability of the world and its sensitivity to our intervention continues to offer an attractive metaphor for artists.

Physicists might rightly complain that this metaphor rests on a misconception. That there is an inherent unknowability about how the future will unfold, and that it might be shifted by almost imperceptible influences, seems far more aptly compared with chaos theory - a purely classical phenomenon, albeit with a quantum equivalent - than with the uncertainty principle. To suggest that Heisenberg's theorem proves we can't acquire perfect knowledge without disturbing that which we seek to understand is, in fact, rather to undersell, as well as to distort, the uncertainty principle.

\section{Uncertainty is a misleading word, implying imperfect knowledge of a state of affairs rather than a fundamentally lacking definition of that state.}

A better way of looking at it is to say that certain pairs of quantum variables cannot meaningfully be said to have simultaneous values defined more tightly than Heisenberg's famous bound of $\hbar / 2$. Uncertainty is a misleading word for that, implying imperfect knowledge of a state of affairs rather than a fundamentally lacking definition of that state. Heisenberg of course expressed it in German in his 1927 paper, talking of both Ungenauigkeit and Unbestimmtheit; translation is inevitably approximate, but these might be reasonably rendered in English as inexactness and undeterminedness. The latter is closer to the mark; the origin of 'uncertainty' might

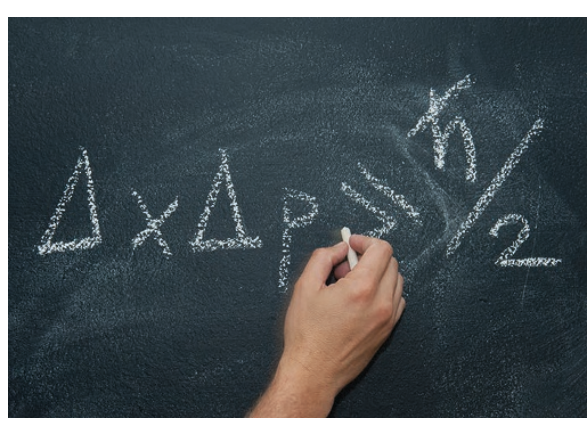

It's debated too whether Heisenberg's somewhat ambiguous discussion of the matter reflected a lack of clarity about the physics or a capitulation to the tenor of the times, when some physicists were influenced by a prevailing febrile sense of crisis and an enthusiasm for antimaterialism. Discussing the uncertainty principle in his 1928 book The Nature of the Physical World, Arthur Eddington felt it opened up a gap in Newtonian determinism that left a space for free will to operate. One might even conclude,

be ascribed to Niels Bohr's preferred term Unsicherheit, which refers to doubtfulness or unsureness.

Heisenberg must carry some responsibility for the confusion, however. Whereas generally he sought to avoid attempts to visualize quantum mechanics, his 1927 paper made that an explicit part of its aim, as the title ('On the visualizable content of quantum theoretical kinematics and mechanics') advertised. And in order to rationalize his uncertainty principle, which followed from noncommutation (a dependence on the order of operation) in his matrix formulation of quantum mechanics, he described a thought experiment using a 'gamma-ray microscope, in which efforts to observe an electron by scattering a single photon from it perturb the path.

The problem with that (quite aside from Heisenberg's shaky grasp of the experimental niceties) is that it implies a precise underlying 'state of affairs' - position and momentum of the electron, say - disrupted by intervention, which is exactly what the uncertainty principle undermines. At root it's a classical argument.

All the same, the analogous uncertainty relationship between error of measurement and magnitude of disturbance that Heisenberg posited was long thought to be basically sound. Only in recent years has it been challenged by the suggestion that the precision of measurement can do better ${ }^{2}-$ an idea prompted originally by attempts to estimate the feasibility of gravitational-wave detection. Opposed points of view, and experimental measurements of the revised error-disturbance relation, have been put forward ${ }^{3-6}$. The upshot is that whether or not you think Heisenberg's relation was correct depends on what you think he meant by it: whether he was talking about one-shot measurements or averages. he said, that "religion first became possible for a reasonable scientific man about the year 1927." The American physicist Percy Bridgman considered that Heisenberg's formula created "a cognitive and moral crisis" - the end, indeed, of the very reach of physics.

Given such drastic interpretations, it was hardly surprising that the uncertainty principle became debased in popular discourse into a kind of existential crisis of modernity: a proof of the limits of knowledge. When the New York Times invoked Heisenberg in 1929 to explain the stock market crash that began the Great Depression, it had its tongue in its cheek; but some religious thinkers were scarcely going to pass on the opportunity Eddington handed them to assert their case.

More than the gap of knowability and predictability, it is Heisenberg's picture of intervention - the observer effect - that seems to speak loudest to modern culture, even though that picture misses the point. The literary critic George Steiner saw an analogy with the way a critical interpretation of a literary text alters its meaning for subsequent generations ${ }^{7}$. Appropriating physics to lend authority - perhaps even a degree of 'proof' - to such (reasonable) observations is not only unnecessary but spurious. It does show, however, that naming matters in science. A catchy slogan can insinuate the most recondite concept into the popular lexicon - for better and worse.

\footnotetext{
References

1. Heisenberg, W. Z. Phys. 43, 172-198 (1927).

2. Ozawa, M. Phys. Rev. A 67, 042105 (2003).

3. Erhart, J. et al. Nat. Phys. 8, 185-189 (2012).

4. Rozema, L. A. et al. Phys. Rev. Lett. 109, 100404 (2012)

5. Busch, P., Lahti, P. \& Werner, R. F. Phys. Rev. Lett. 111, 160405 (2013).

6. Ringbauer, M. et al. Phys. Rev. Lett. 112, 020401 (2014)

7. Crease, R. P. \& Goldhaber, A. S. The Quantum Moment 154 (W. W. Norton, 2014)
} 